\title{
Parameter Optimization Of CPG And Its Application In Robot
}

\author{
Qiang $\mathrm{Lu}^{\mathrm{a}}$, Zhaochen Zhang ${ }^{\mathrm{a}}$ and Wenfeng Li \\ College of Medical Information and Engineering, Taishan Medical University, Taian, 271016, China
}

\begin{abstract}
The central pattern generator $(\mathrm{CPG})$ has been identified in the spinal cords as responsible for locomotion movements. It is difficult to select the appropriate values of parameters in CPG model. In this paper, the Matsuoka oscillator is selected as the rhythm-generation model and the bat algorithm is chosen to search the parameters of CPG model. The paper shows the details of the parameters optimization and the diagram of the convergence performance. In the paper, the CPG includes the main rhythm-generation neuron and the minor pattern-formation neuron, and Rowat's neural model is chosen as the patternformation neuron. Then the CPG model is applied to the three-link biped robot and the result shows that the method of parameter optimization is effective.
\end{abstract}

\section{Introduction}

The CPG refers to the biological neuronal circuit that exists within the spinal cord of the vertebrate [1]. A CPG is a group of neurons interconnected in a specific manner, which can produce rhythmic outputs to activate muscle contractions, resulting in coordinated rhythmic body motions $[2,3]$. Many researchers have studied the CPG in the fields of robot control [4] and the modeling [5]. In order to represent the CPG and generate the required signals, several nonlinear oscillators that are coupled together have been developed, such as the Rayleigh, Wilson-Cowan, Kuramoto, Van del Pol, Matsuoka oscillator and multi-layered multi-pattern CPG model [2, $6]$.

In CPG model, many parameters are needed to be set, such as the time parameter and the parameter of the inhibitory connection. It is difficult to select the appropriate values of parameters in CPG model. The researchers found that the swarm intelligence algorithms are effective methods to solve the parameters optimization. Kim et al. [7] used nonparametric estimation based particle swarm optimization to search the parameters of CPG. In the swarm intelligence algorithms, the bat algorithm is a new algorithm and it is applied in many optimization problems[8, 9]. The researchers have also proved that the bat algorithm is better than many other algorithms [10]. In this paper, the bat algorithm is used to optimize the parameters of the CPG model.

Grillner [11] has conformed the locomotion control system. The CPG produce motor patterns with appropriate timing. The generator is affected by sensory feedback and is activated from the brainstem locomotor command regions. Lacquaniti et al. [12] shown that muscle activity is accounted for by a combination of few basic patterns, each one timed at a different phase of the gait cycle. The corresponding weights of distribution to different muscles may change as a function of the condition, allowing highly flexible control. In this paper, a new CPG model is established, which includes the main rhythm-generation neuron and the minor patternformation neuron. The Matsuoka oscillator and cell model of Rowat are selected as the rhythm-generation model and pattern-formation model respectively. Based on previous locomotion control model[4], the new CPG model is applied into the robot locomotion.

This paper is organized as follows. Section 2 presents the parameters optimization of the CPG model. The simulations are shown in Section 3. The conclusions and future works are made in Section 4.

\section{Parameters optimization of the CPG model}

In this paper, the Matsuoka model $[14,15]$ is chosen as the CPG model. The Matsuoka model can be described by.

$$
\left\{\begin{array}{l}
T_{r} \dot{u}_{1}+u_{1}=-d u_{3}-w g\left(u_{2}\right)+e \\
T_{r} \dot{u}_{2}+u_{2}=-d u_{4}-w g\left(u_{1}\right)+e \\
\quad \dot{u_{3}}+u_{3}=g\left(u_{1}\right) \\
\quad \dot{u_{4}}+u_{4}=g\left(u_{2}\right) \\
v_{1}=g\left(u_{1}\right)
\end{array}\right.
$$

The function $g(\bullet)$ is a piecewise linear function defined by $g(u)=\max (o, u)$, which represents a threshold property of the neurons. The variables $u_{1}, u_{3}$ and $v_{1}$ represent the membrane potential and the firing rate of the neuron, respectively. Self-inhibitory input $u_{2}$ and $u_{4}$ represent an adaptation or fatigue property that

a Corresponding authors. Email: luqiang271016@163.com and zc.zh@163.com 
ubiquitously exists in real neurons. The parameter $e$ denotes the tonic input. Parameters $w$ and $d$ represent the strength of mutual and self inhibition, respectively. Parameters $T_{r}$ and $T_{a}$ are the time constants that determine the reaction times of variables $u_{1}, u_{3}$ and $u_{2}, u_{4}$

The CPG model has applied into the robot locomotion control[4]. In application, the parameter optimization is a problem which need to be solved. The researchers[7, 13] have used particle swarm optimization and genetic algorithms to search the parameters of CPG. Then the swarm intelligence algorithms are effective methods to solve this problem.

The bat algorithm is one of the recent bio-inspired optimization techniques. The bat algorithm uses the echolocation behavior for sensing distance and to differentiate between prey and obstacles. The bats emit a very loud sound and use the time delay from surroundings to detect the around objects. The intervention between the bounced and the reflected pulses helps the bats to decide the obstacle type. The bat algorithm $[8,9]$ is described by.

$$
\left\{\begin{array}{l}
f_{i}=f_{\text {min }}+\left(f_{\text {max }}-f_{\text {min }}\right) \times \beta \\
v_{i}^{t}=v_{i}^{t-1}+\left(x_{i}^{t}-x_{*}\right) f_{i} \\
x_{i}^{t}=x_{i}^{t-1}+v_{i}^{t}
\end{array}\right.
$$

where $\beta$ is a random number in the range $[0,1]$ drawn from the uniform distribution. $x_{*}$ is the current global best location. $f_{\min }$ and $f_{\max }$ represent the minimum and maximum frequency, respectively. And $v_{i}$ represents the velocity vector. In the bat algorithm, the positions $x_{i}$ of bats are randomly initialized in the search space. The number of bats is determined by the user. The positions of the bats are adjusted by adding a velocity to the old positions. The velocity of bats is also randomly initialized and it can be calculated.

Then the flow diagram of the bat algorithm is shown in Figure 1.

In the flow diagram of the bat algorithm, when rand $>r_{i}$, a new solution for each bat is generated by.

$$
x_{\text {new }}=x_{\text {old }}+\mathcal{E} A^{t}
$$

where $\varepsilon$ ia a random number in the field $[-1,1] . A^{t}$ is the average loudness of all the bats.

When $r a n d<A_{i}$ and $F($ new $)<F\left(x_{i}\right)$, the variables $r_{i}$ and $A_{i}$ are updated by.

$$
\left\{\begin{array}{l}
A_{i}^{t+1}=\alpha A_{i}^{t} \\
r_{i}^{t+1}=v_{i}^{0}[1-\exp (-\gamma t)]
\end{array}\right.
$$

where $\alpha$ and $\gamma$ are constant.

In the Matsuoka model, there are four parameters which are needed to be set. They are $T_{r}, T_{a}, w$ and $d$. According to reference [16], the parameters are set as $T_{a}=10 T_{r}$ and $w=d$. Then the bat algorithm is used to optimize the parameters $T_{a}$ and $w$. The regions of $w$ and $T_{a}$ are set as[2, 4] and $[0.1,2]$, respectively. In the bat algorithm, the number of iterations is 500 . The population size is 100 and the number of variables for optimization is 2. And other parameters are set as $\alpha=\gamma=0.95, f_{\min }=0$ and $f_{\max }=2$.

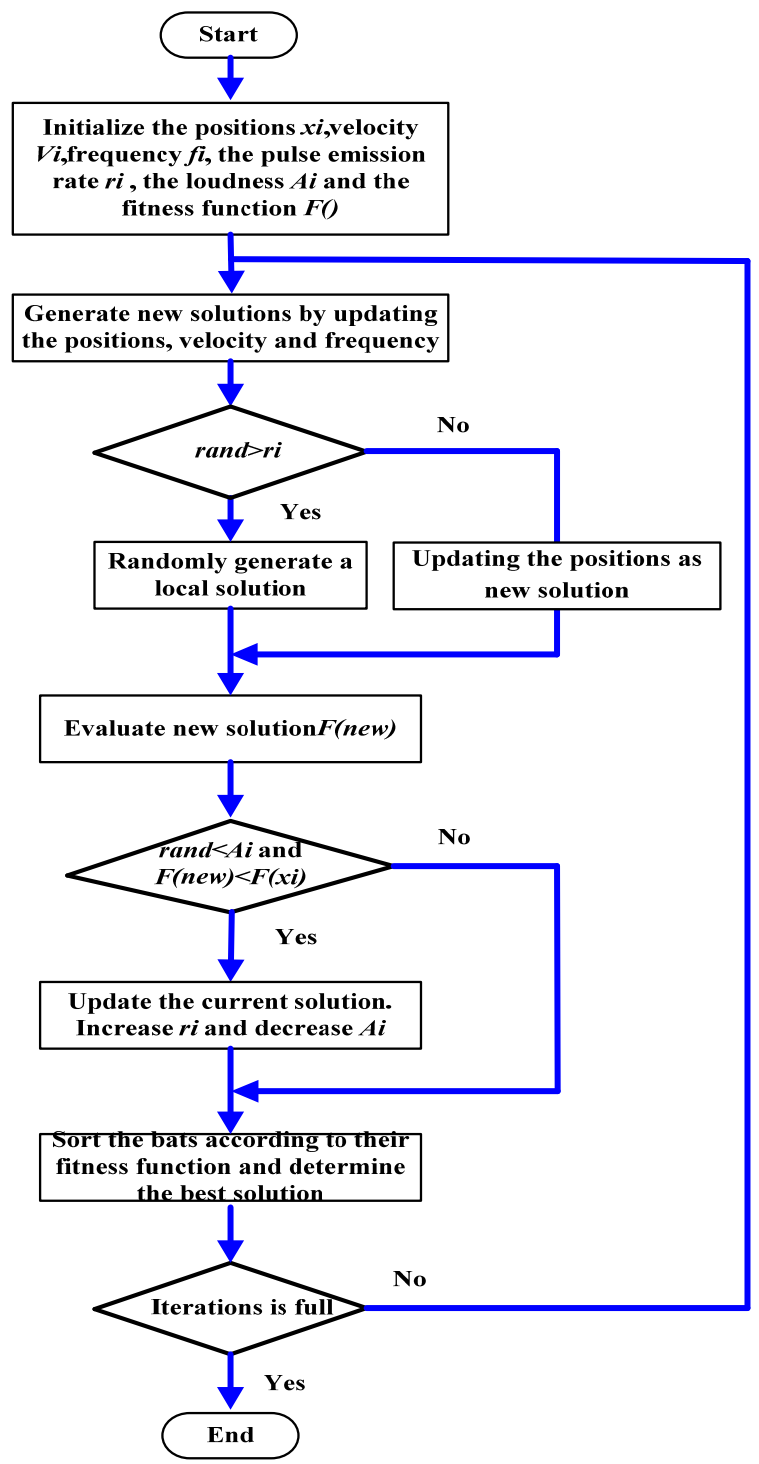

Figure 1. The flow diagram of bat algorithm.

In the parameter optimization, the fitness function is selected as.

$$
f i t=\frac{\sum_{j=1}^{n}\left(C P G_{\text {out }}-M_{\text {out }}\right)}{n}
$$

where $C P G_{\text {out }}$ is the output of the CPG model. $M_{\text {out }}$ is the CPG output according to the reference [4]. Parameter $n$ is the population size.

Based on the flow diagram of parameter optimization, the values of parameters are obtained. They are $T_{a}=0.2$, $w=d=2.5$. The convergence performance of parameter $T_{a}$ is shown in Figure 2.

In Figure 2. the parameter value converge into the 0.2 after iterative computation. 


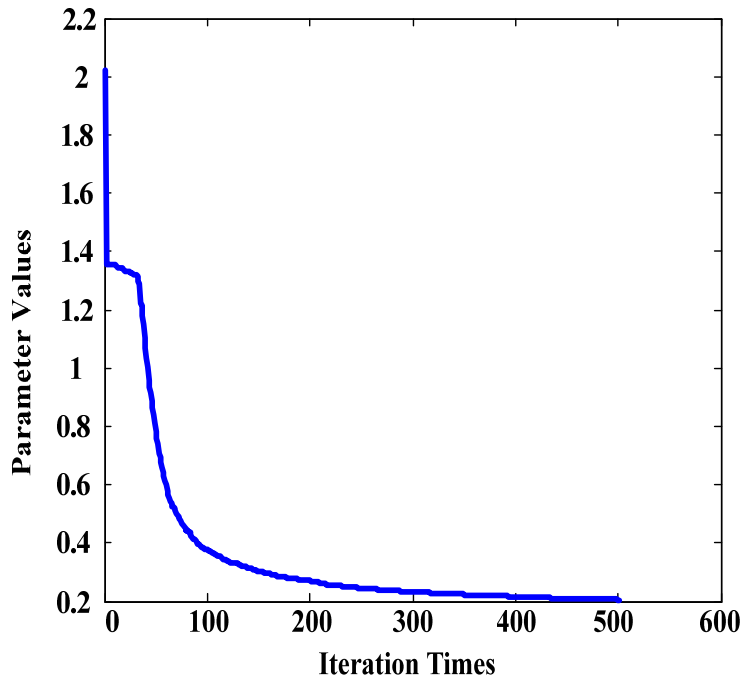

Figure 2. Convergence performance of the parameter optimization.

\section{Application in robot and simulations}

The three-link biped robot [17] is employed to simulate cyclic walking gait. The model is expressed in Figure 3.

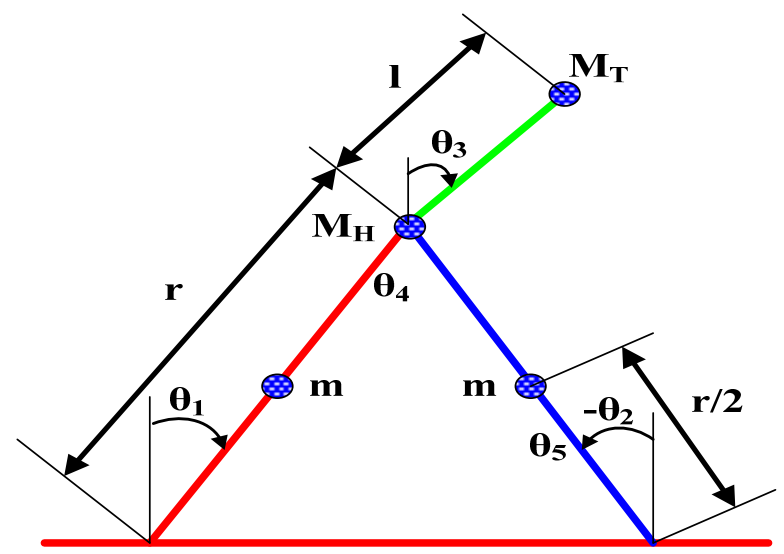

Figure 3. Model of three-link biped robot.

The biped model consists of three links, namely the torso and two links in each leg. In this model, all masses are lumped. The legs are symmetric, with length $r$, and the mass of each leg is lumped at $r / 2$. The distance from the hips to the center of mass of the torso is denoted by 1 . The model is indicated in a set of absolute coordinates, that is $\theta_{1}, \theta_{2}$ and $\theta_{3}$ are each referenced with respect to the inertial frame. The mass of torso, hip and leg are $M_{T}$, $M_{H}$ and $m$, respectively. In following simulation, these parameters are set as $l=0.5 \mathrm{~m}, \quad r=1 \mathrm{~m}, \quad M_{T}=10 \mathrm{~kg}$, $M_{H}=15 \mathrm{~kg}$ and $m=5 \mathrm{~kg}$ [18].

Rowat et al. [20] proposed a neural model based on two cells with self-rhythmic generation ability. Each cell independently generates its own pattern according to two parameters that are related to the membrane conductivities for fast and slow currents. All fast membrane currents are represented by a single fast current, and all slow membrane currents are represented by a single slow current. This model is represented by two differential equations.

$$
\left\{\begin{array}{l}
\tau_{m} \frac{d V}{d t}=-\left(\operatorname{fast}\left(V, \sigma_{f}\right)+q-i_{i n j}\right. \\
\tau_{s} \frac{d q}{d t}=-q+q_{\infty}(V)
\end{array}\right.
$$

where $V$ is the membrane potential, and $q$ is the lumped slow current. While the fast current is supposed to activate immediately, the membrane time constant $\tau \mathrm{m}$ is assumed to be significantly smaller than the slow current's time constant for activation $\tau_{s}$. The injected current is $i_{i n j}$ and $\sigma_{f}$ is shape parameter for the currentvoltage curve . An idealized current-voltage curve for the lumped fast current is given by the following equation.

$$
\left(\operatorname{fast}\left(V, \sigma_{f}\right)=V-A_{f} \tanh \left(\left(\sigma_{f} / A_{f}\right) V\right)\right.
$$

The steady-state value of the lumped slow current is linear in $V$ with conductance $\sigma$ s, which is given by.

$$
q_{\infty}(V)=\sigma_{s} V
$$

In this model, with different combinations values of $\sigma_{s}$ and $\sigma_{f}$, the neuron shows different modes of activity [2].

The researchers[2, 12] have found that the $\mathrm{CPG}$ includes the main rhythm-generation neuron and the minor pattern-formation neuron. In following simulation, Rowat's neural model is chosen as the pattern-formation neuron. The sum of the Matsuoka oscillator and cell model of Rowat are selected as the CPG signal.

Taga et al. [21] showed the basic structure of human locomotion. The previous study [4] has established a modified locomotion control model based on Taga model. In the modified model, the CA-CMAC is selected to replace the motor command generation and impedance controller and used to realize the pattern mapping between the CPG output and the musculoskeletal system. The outputs of the CPG network are changed into the positions and angles inspiring the musculoskeletal system and the walking gait is then obtained. In the paper, the CPG is replaced by the the Matsuoka oscillator and cell model of Rowat. The modified locomotion model is shown in Figure 4.

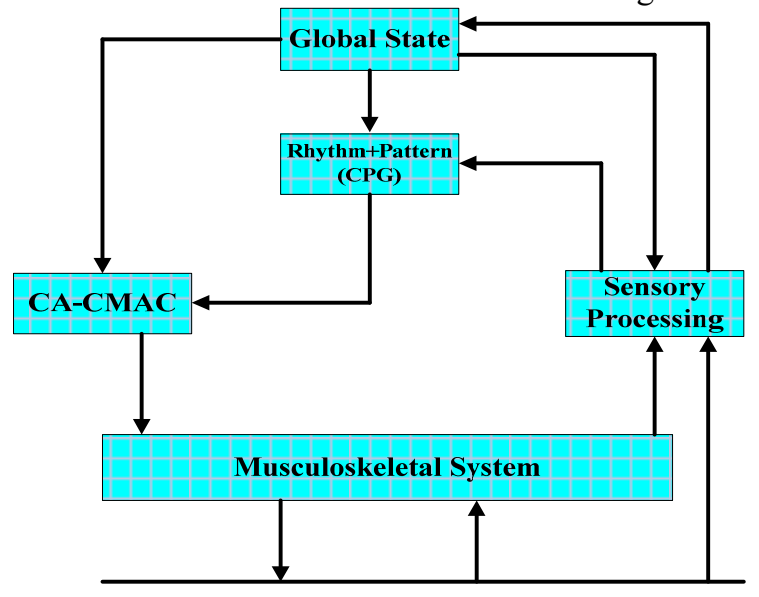

Environment

Figure 4. The locomotion control model.. 
When the CPG is applied in robot, the CPG oscillators are allocated at each joint and its output is used as joint position commands to the robot. Desired gait is generated by coordinating the movement of multiple oscillators in a self-organizing manner [19]. In this paper, the topology of the CPG network is annular and these adjacent CPGs are coupled to one another.

In following simulation, one cycle outputs of $\mathrm{CPG}$ corresponding to one walking cycle angles and the sampling size is 400 . The input values of CA-CMAC[4] are two-dimension. One is the sampling values of CPG, and the other is the differential of these values. Three joints' angles are needed to be converted in simulation.

The output of CPG and data collected based on studies in [17] are chosen as the input values and expected values of the CA-CMAC, respectively. It assumes that the walking step is five. Therefore, ten cycles of data are collected. The first five cycles data are used to train CA-CMAC and the other values are used to test. The expected angles $\left(\theta_{1}, \theta_{2}, \theta_{3}\right)$ are shown in Figure 5 .

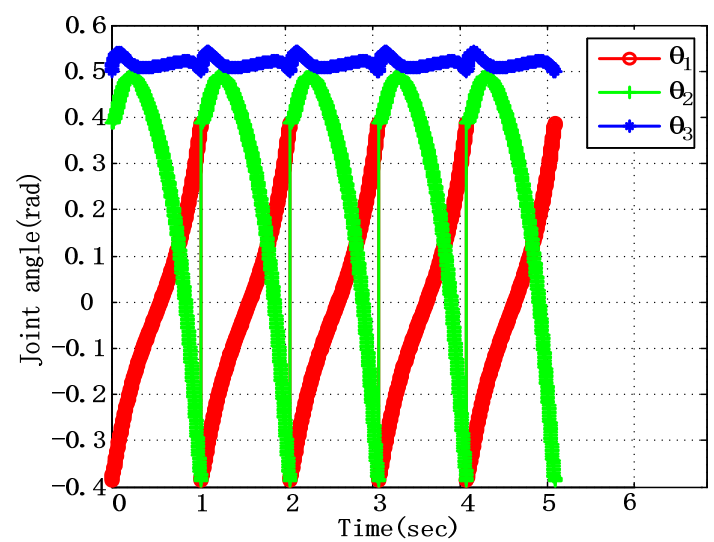

Figure 5. The expected angles.

Based on the test data of CA-CMAC, the stick figures of the walking movement are expressed in Figure 6.

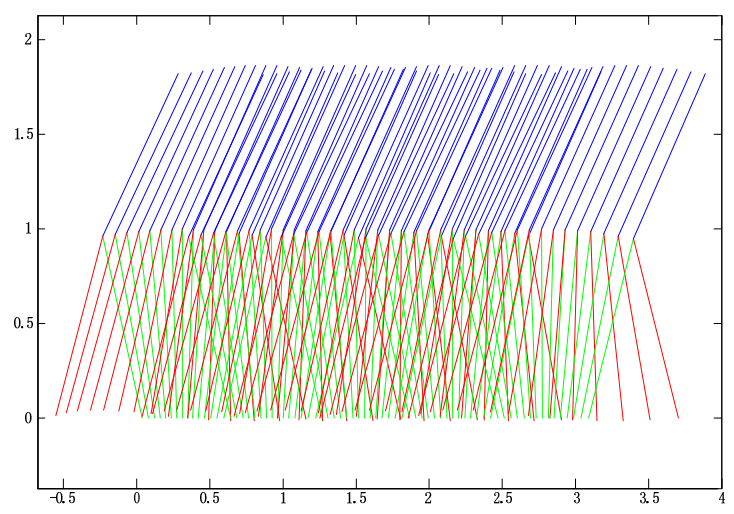

Figure 6. The expected angles

In Figure 6, the green, red and blue lines represent the stance leg, the swing leg and the torso, respectively. The results show that the CPG model is effective.

\section{Conclusions}

In this paper, the Matsuoka oscillator is selected as the rhythm-generation model and the bat algorithm is chosen to search the parameters of CPG model. The paper shows the details of the parameters optimization and the diagram of the convergence performance. Then the $\mathrm{CPG}$ model is applied to the three-link biped robot and the result shows that the method of parameter optimization is effective.

Recently, an idea which is usually termed embodiment is studied by many researchers [22]. The research shows that the locomotion control is not only located in the brain, but that there is a tight coupling among the brain, the body, and the environment. The embodiment can reduce the burden of the brain and make the control easy and is deserved to investigate further.

\section{Acknowledgement}

The work was supported by Shandong Provincial Natural Science Foundation, P. R. China under Grant No. ZR2017MF039.

\section{References}

1. K. J. Dougherty, L. Zagoraiou, D. Satoh, et al., Neuron 80, 14 (2013)

2. J. Yu, M. Tan, J. Chen, et al., IEEE Transactions on Neural Networks and Learning Systems 25, 16 (2014)

3. M. N. Islam, Z. Chen, IEEE Transactions on Robotics 30, 15 (2014)

4. Q. Lu, J. Tian, Mathematical Problems in Engineering 2015, 9 (2015)

5. I. A. Rybak, N. A. Shevtsova, M. Lafreniere-Roula, et al., The Journal of physiology 577, 23 (2006)

6. G. L. Liu, M. K. Habib, K. Watanabe, et al., Artificial Life and Robotics 12, 6 (2008)

7. J. J. Kim, J. W. Lee, J. J. Lee, International Journal of Control, Automation, and Systems 7, 11 (2009)

8. A. Tharwat, A. E. Hassanien, B. E. Elnaghi, Pattern Recognition Letters 93, 10 (2017)

9. A. Chakri, R. Khelif, M. Benouaret, et al., Expert Systems with Applications 69, 17 (2017)

10. G. Wang, L.Guo, Journal of Applied Mathematics 2013, 16 (2013)

11. S. Grillner, Science 334, 2 (2011)

12. F. Lacquaniti, Y. P. Ivanenko, M. Zago, Journal of Physiology 590, 11 (2012)

13. T. Reil, P. Husbands, IEEE Transactions on Evolutionary Computation 6, 10 (2002)

14. Q. Lu, Cognitive Neurodynamics 9, 14 (2015)

15. Q. Lu, J. Tian, Cognitive Neurodynamics 8, 10 (2014)

16. K. Matsuoka, Biological Cybernetics 104, 8 (2011)

17. J. W. Grizzle, G. Abba, F. Plestan, IEEE Transactions on Automatic Control 46, 14 (2001)

18. E. R. Westervelt, J. W. Grizzle, C. Chevallereau, et al., UK: Taylor \& Francis Group 17 (2007) 
19. J. J. Kim, J. J. Lee, International Conference on Control, Automation and Systems 19, 6 (2007)

20. P. F. Rowat, A. I. Selverston, Journal of Computational Neuroscience 4, 25 (1997)

21. G. Taga, Biological Cybernetics 73, 15 (1995)

22. K. Nakajima, H. Hauser, R. Kang, et al., Frontiers in Computational Neuroscience 7, 9 (2013) 